\title{
Vascular Access Surveillance: Current Concepts
}

\section{Konstantinos Leivaditis and Vassilios Liakopoulos* \\ $1^{\text {st }}$ Department of Internal Medicine, Division of Nephrology and Hypertension, AHEPA Hospital, School of Medicine, Aristotle University of Thessaloniki, Greece}

*Corresponding author: Vassilios Liakopoulos, Assistant Professor of Nephrology, Division of Nephrology and Hypertension, $1^{\text {st }}$ Department of Internal Medicine, AHEPA Hospital, School of Medicine, Aristotle University of Thessaloniki, 1, St. Kyriakidi street, 54636, Thessaloniki, Greece, Tel: +302310994694; E-mail: liakopul@otenet.gr

Rec date: Jul 11, 2014, Acc date: Jul 15, 2014, Pub date: Jul 18, 2014

Copyright: @ 2014 Leivaditis K, et al. This is an open-access article distributed under the terms of the Creative Commons Attribution License, which permits unrestricted use, distribution, and reproduction in any medium, provided the original author and source are credited.

\section{Description}

Vascular access (VA) remains a major contributing factor to the morbidity and mortality of hemodialysis (HD) patients. More than $30 \%$ of the hospitalizations of long term hemodialysis patients in the United States are related to VA and approximately $\$ 1$ billion is spent annually to manage VA complications [1,2]. A well-functioning VA is a matter of critical importance for the quality of life of these patients and it is strongly associated with adequate dialysis and prolonged technique and patient survival. However, it still remains the Achille's heel of HD.

The development of stenosis is the major factor that leads to VA dysfunction and can eventually lead to thrombosis. It is therefore reasonable to devise strategies for early detection of lesions within a VA system before serious complications arise. This could be accomplished by performing surveillance and monitoring. The former refers to the periodic evaluation of VA by using tests that may involve specific instrumentation. Access monitoring is a clinical procedure and refers to physical examination of the VA to detect physical signs that suggest the presence of dysfunction. It is obvious that surveillance and monitoring are complementary [3].

The Dialysis Outcome Quality Initiative guidelines suggest that HD units should implement surveillance programs for arteriovenous fistulae (AVF) and grafts (AVG). These programs should aim at prompt detection of VA dysfunction and timely referral for correction, reduction of central venous catheter use and decrease of hospitalization and overall cost.

Suggested surveillance methods for both AVF and AVG include access blood flow (ABF) measurement, static pressure evaluation and duplex ultrasonography. Access recirculation (not urea based) and dynamic pressure measurements are accepted methods for AVF. Physical examination is an accepted method in contrast to nonstandardized dynamic pressure measurement for AVG. Decreasing URR or $\mathrm{Kt} / \mathrm{V}$ (otherwise unexplained) and increased (negative) arterial pressure in the dialysis machine are methods of limited sensitivity and specificity for both AVF and AVG [1].

Measurement of $\mathrm{ABF}$ has been proposed as the gold standard for the screening of both AVF and AVG. ABF can be measured by a variety of techniques which are direct or indirect. Direct methods include duplex Doppler ultrasonography and magnetic resonance angiography. Indirect methods such as ultrasound dilution (Transonic), optodilutional (Critline) or conductivity (GambroFresenius) are performed during the hemodialysis session [4]. At present, the optimal blood flow threshold to trigger referral for angiographic imaging (fistulography) in AVF has not been determined. The thresholds for AVG (flow $<600 \mathrm{~mL} / \mathrm{min}$ or a $25 \%$ reduction in flow if flow $<1000 \mathrm{~mL} / \mathrm{min}$ over 4 months) may not be appropriate, especially given that AVF remain patent at lower levels of blood flow.

According to the latest DOQI guidelines, prospective surveillance of fistulae and grafts for hemodynamically significant stenosis, when combined with correction of the anatomic stenosis, may improve patency rates and may decrease the incidence of thrombosis [1]. We should bear in mind that besides stenosis, hypotensive episodes, external compression, improper cannulation or hypercoagulability can also lead to thrombosis.

Several studies about VA surveillance programmes have been performed and most of them failed to demonstrate its utility and necessity while other have emphasized a beneficial effect on VA survival. Therefore, the role of surveillance remains unproved and somewhat controversial. An elegant review by Paulson et al highlights this controversy about the necessity and the role of VA surveillance and concludes that current published data is insufficient to support the concept that all accesses should undergo routine screening with intervention [5]. The same authors advocate the recommendation of a vascular access maintenance program without recommending specific surveillance measurements with preemptive intervention [6]. They also argue that VA surveillance controversy provides a case study of how the excessive enthusiasm for a new test or treatment can lead to the adoption of a false paradigm. Paradigms are the beliefs and assumptions shared of those in a field of knowledge and are commonly incorporated in clinical practice guidelines. According to them, VA surveillance should not be currently recommended or performed in a routine basis and that it promotes the treatment of the identified stenosis, usually by angioplasty without any proven benefit. Furthermore, multiple angiographic interventions may deteriorate things and promote further stenosis by enhancing neointimal hyperplasia [7,8]. A recent analysis by Tessitore et al also comments that VA surveillance has been described as an example of how new tests are sometimes adopted even without good-quality evidence of their advantages. However they claim that this may be true for AVG but not necessarily for AVF. Their analysis strongly suggests that ABF surveillance is an effective screening tool for mature AVF although further studies are needed to clarify its benefits and cost effectiveness [9].

Despite the conflicting data, nobody seems to disagree that clinical monitoring by physical examination is a cost effective, simple and validated bedside tool to detect access dysfunction that can be easily taught and performed by everyone from a novice to an expert [10]. Several studies have confirmed the value of physical examination in accurately detecting both inflow and outflow stenosis in an AVF with $85-90 \%$ sensitivity and $75-80 \%$ specificity [11-13]. The findings can be limited in case of concurrent inflow and outflow stenosis. 
Citation: Konstantinos Leivaditis and Vassilios Liakopoulos (2014) Vascular Access Surveillance: Current Concepts. J Nephrol Ther 4: 1000e110. doi:10.4172/2161-0959.1000e110

Page 2 of 2

In the second decade of the 21st century, VA morbidity still haunts the nephrology community. There remains a great need for properly designed randomized clinical trials which would shed light to basic unanswered questions in the field of VA research. Indiscriminate and routine performance of surveillance testing alone should not be mandated and is not currently recommended. Until solid evidence is available to entirely depend on surveillance techniques, we must ensure that everyone involved in the management of HD patients is trained adequately to physically examine a VA.

\section{References}

1. National Kidney Foundation (2006) K/DOQI clinical practice guidelines in vascular access: 2006 update. Am J Kidney Dis 48[Suppl 1]: S176S306.

2. US Renal Data System: Annual Data Report: Atlas of Chronic Kidney Disease and End-Stage Renal Disease in the United States, Bethesda, National Institutes of Health, National Institute of Diabetes and Digestive and Kidney Diseases, 2007.

3. Besarab A (2006) Access monitoring is worthwhile and valuable. Blood Purif 24: 77-89.

4. Leivaditis K, Panagoutsos S, Roumeliotis A, Liakopoulos V, Vargemezis V (2014) Vascular access for hemodialysis: postoperative evaluation and function monitoring. Int Urol Nephrol 46: 403-409.

5. Paulson WD, Moist L, Lok CE (2012) Vascular access surveillance: an ongoing controversy. Kidney Int 81: 132-142.
6. Paulson WD, Moist L, Lok CE (2013) Vascular access surveillance: case study of a false paradigm. Semin Dial 26: 281-286.

7. Moist LM, Churchill DN, House AA, Millward SF, Elliott JE, et al. (2003) Regular monitoring of access flow compared with monitoring of venous pressure fails to improve graft survival. J Am Soc Nephrol 14: 2645-2653.

8. Chang CJ, Ko PJ, Hsu LA, Ko YS, Ko YL, et al. (2004) Highly increased cell proliferation activity in the restenotic hemodialysis vascular access after percutaneous transluminal angioplasty: implication in prevention of restenosis. Am J Kidney Dis 43:74-84.

9. Tessitore N, Bedogna V, Verlato G, Poli A (2014) The rise and fall of access blood flow surveillance in arteriovenous fistulas. Semin Dial 27: 108-118.

10. Vachharajani TJ (2012) Diagnosis of arteriovenous fistula dysfunction Semin Dial 25: 445-450.

11. Campos RP, Chula DC, Perreto S, Riella MC, do Nascimento MM (2008) Accuracy of physical examination and intra-access pressure in the detection of stenosis in hemodialysis arteriovenous fistula. Semin Dial 21: 269-273.

12. Asif A, Leon C, Orozco-Vargas LC, Krishnamurthy G, Choi KL, et al. (2007) Accuracy of physical examination in the detection of arteriovenous fistula stenosis. Clin J Am Soc Nephrol 2:1191-1194.

13. Coentrão L, Faria B, Pestana M (2012) Physical examination of dysfunctional arteriovenous fistulae by non-interventionalists: a skill worth teaching. Nephrol Dial Transplant 27: 1993-1996. 